\title{
Synthesis and characterization of new Hemorphin-5 analogue containing azobenzene moiety with potential optical switching properties
}

\author{
Petar Todorov, Petia Peneva, Stela Georgieva-Kiskinova, Anton Georgiev \\ University of Chemical Technology and Metallurgy, Sofia, 1756, blvd.Kliment Ohridski 8, Bulgaria
}

https://doi.org/10.17952/35EPS.2018.121

\section{Introduction}

The discovery of bio molecular control of pharmacophores and their macromolecular motion by light offers dynamic changing of the properties of molecules in biological systems with minimal disturbance to the rest of the system. Azobenzene containing peptides could be acting as bio photoswitches that interconvert between E- and $\mathrm{Z}$ - isomers around $-\mathrm{N}=\mathrm{N}$ - group offer highly predictable light state structural properties. These molecules are relatively small and adopt predictable conformations they are well suited as tools to interrogate cellular function in a spatially and temporally controlled fashion and for applications in photopharmacology and photodynamic therapy $[1,2]$. Photodynamic control of peptides, proteins or drugs provides a non-invasive way to disorder these networks to investigate their effect or cause a defined outcome.

In order to evaluate the influence of azobenzene-peptides we introduced the azobenzene-4,4'-dicarboxylic acid to the $\mathrm{N}$-side of Hemorphin-5 peptide analogue. This report refers to the solid-phase synthesis and characterization of novel azobenzene containing Hemorphin-5 analogue. Hemorphin-5, also known as Valorphin, is a naturally occurring, endogenous opioid peptide of the Hemorphin family with affinity for opioid receptors and morphinomimetic properties. These neuropeptides are increasingly being used in the treatment of various diseases such as hypertension, epilepsy, chronic pain, cancer, and etc. $[3,4]$.

\section{Results and Discussion}

By considering, the scarce information about the synthesis of azo-peptides, and especially their $\mathrm{E} \rightarrow \mathrm{Z}$ isomerization proves the challenging of preparation and characterization such compounds. The investigation of the electrochemical oxidation and reduction mechanisms of these compounds is important since it could result in a better understanding of their physiological mechanisms in order to receive new information on biomolecular ox-red level. To assess the redox properties of azobenzene moiety in Valorphin, and to investigate the $\mathrm{E} \rightarrow \mathrm{Z}$ conversion, the cyclic and differential pulse voltammetry at Pt- working electrode was used.

The synthesis was achieved by a modified solid-phase peptide synthesis by Fmoc-(9-fluorenylmethoxy-carbonyl) dimerization strategy, based on the reaction of azobenzene-4,4'-dicarboxylic acid with two $\mathrm{N}$-terminalamino groups of distinct Val-Val-Tyr-Pro-Trp-Thr-Gln heptapeptides directly on the resin (Fig. 1) to (Val-Val-Tyr-ProTrp-Thr-Gln) ${ }_{2}$ Azo peptide.

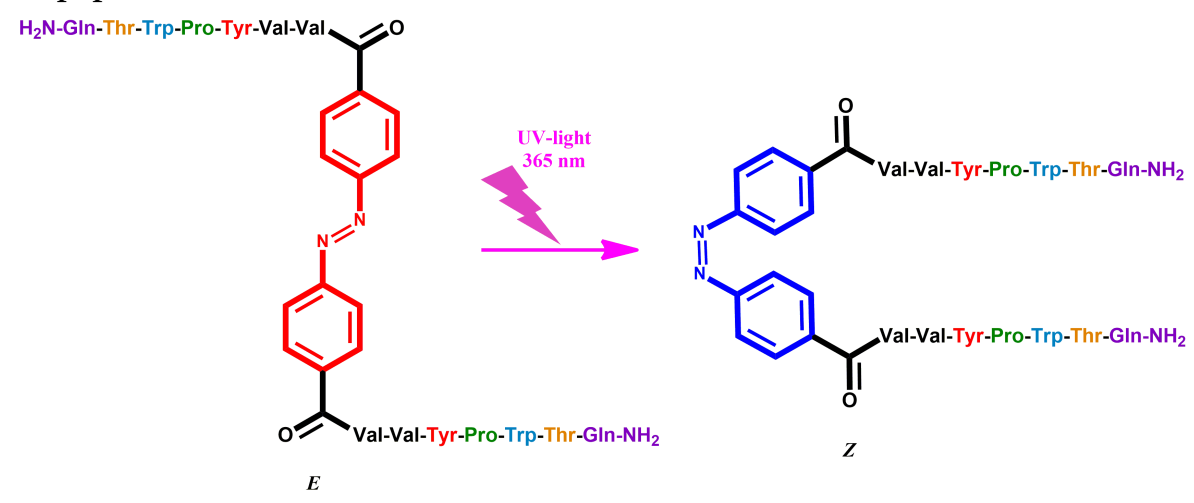

Figure 1: Schematic representation of photoswitchable (Val-Val-Tyr-Pro-Trp-Thr-Gln) ${ }_{2}$ Azo peptide upon long wavelength UV light as trans $(E)$ and cis $(Z)$ isomers. 
For proving that obtained results are for azo-peptide only and to be investigate the electrochemical behaviour of such interesting azo-compound on the electrode surface the cyclic and differential pulse voltamperometry on Pt-electrode were examined. Several processes (such as oxidations and reductions) can be identified by direct analysis of the cyclic voltammograms on Pt-electrode (Fig.2). However, as previous studies have proved, it is essential to consider a working-window analysis to differentiate azo-peptide related redox processes from those of secondary reactions on azobenzene-4,4'-dicarboxylic acid and VV-hemorphin-5and among other misleading interferences. The electrochemical study of Valorphine and Valorphine derivatives were previously reported by Todorov et al. 2018 [5]. Anodic cyclic voltammogram for the oxidation of VV-hemorphin-5in phosphate buffer solution at Pt- electrode is shown in Fig. 2. The anode peaks of the compounds are probably due to the presence of tryptophanin the peptide molecule, which is oxidized to a platinum electrode at potential closed to $+0.4 \mathrm{~V}$ [6]. The results on the voltammogram exhibits one anodic and cathodic peaks at 193 and $-113 \mathrm{mV}$, respectively, which shows a quasi- reversible behaviour, with peak-to-peak separation $(\Delta \mathrm{Ep}=\mid$ Epc-Epa $\mid)$ of $80 \mathrm{mV}$ and it is proves the theoretical obtained data. At the same condition the voltammogramsof rest compounds were recorded (Fig. 2). The reduction potentials and nature of electrodic processes depend on the UV-irradiation of azo-peptide with $\lambda=365 \mathrm{~nm}$ and while the reduction potential have a value near to zero and quasi-reversible reduction for $\mathrm{AzP}(\mathrm{N})$ it was observed that $\mathrm{AzP}(\mathrm{IRr})$ have a more negative potential value and fully irreversible reduction (Fig. 2). The data were confirmed using DPV mode. Two well defined oxidation peaks for AzP before irradiated were observed in DPV mode; one at more negative potential and second at $\mathrm{Ep}_{\mathrm{a}}=0.300 \mathrm{~V}$ corresponding to $-\mathrm{N}=\mathrm{N}$-group and VV-hemorphin-5, respectively (Fig. 3).

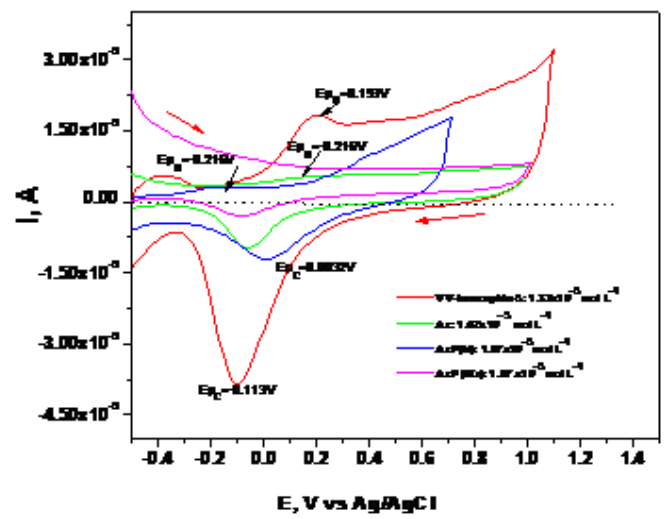

Figure 2: Cyclic voltammograms of azobenzene-4,4'-dicarboxylic acid (Az), VV-hemorphin-5 and VV-hemorphin5 azopeptide $(A z P)$ before $(A z P(N))$ and after $(A z P(I R r))$ illumination at $\lambda=365 \mathrm{~nm}$ whit same concentrations in pH 6.86 (phosphate buffer solution, $0.1 \mathrm{~mol} \mathrm{~L}^{-1}$ ), scan rate $1.00 \mathrm{~V} \mathrm{~s}^{-1}$, with working Pt-and $\mathrm{Ag} / \mathrm{AgCl}, \mathrm{KCl}$ as reference electrodes.

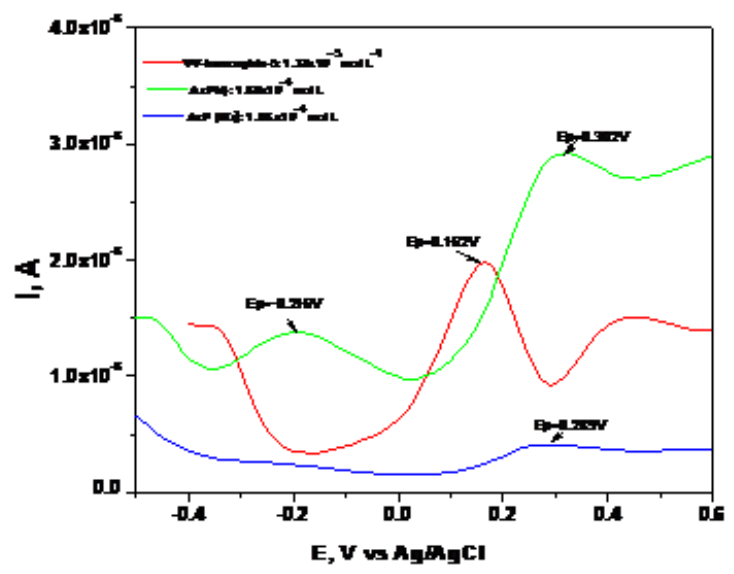

Figure 3: DPVs of VV-hemorphine-5 and azo-peptide $(A z P)$ before and after UV irradiated at $\lambda=365 \mathrm{~nm}$ in $\mathrm{pH}$ 6.86 (phosphate buffer solution, $0.1 \mathrm{~mol} \mathrm{~L}^{-1}$ ) at Pt- and $\mathrm{Ag} / \mathrm{AgCl}, \mathrm{KCl}$ as reference electrodes. 


\section{Conclusions}

We describe the synthesis and electrochemical properties of new analogue of Hemorphin- 5 containing azobenzene moiety. The compound was obtained by solid-phase peptide synthesis - Fmoc-dimerization strategy. The electrochemical properties are discussed and confirm in order to the $\mathrm{E} \rightarrow \mathrm{Z}$ transformation changes on electrochemical behaviour of the compound by altering the reactivity of the redox function group.

\section{Acknowledgments}

This work was financial supported by the Bulgarian National Scientific Fund project 18/5 of the Ministry of Education and Science, Bulgaria.

\section{References}

1. Mart RJ, Allemann RK. Chem. Commun. 52:12262 (2016).

2. Renner C, Moroder L. ChemBioChem 7:869 (2006).

3. Blishchenko E, Sazonova O, Kalinina O, Yatskin O, Philippova M, Surovoy A, Karelin A, Ivanov V. Peptides 23:903-910 (2002).

4. Song CZ, Wang WQ, Song CC. Int. J. Cancer 131:1011-1012 (2012).

5. Todorov P, Peneva P, Pechlivanova D, Georgieva S, Dzhambazova E. Bioorg. Med. Chem. Lett. 28:3073-3079 (2018).

6. MacDonald SM, Roscoe SG. Hecfrochimico Acta 42:1189-1200 (1997). 